\title{
Combining Discriminant and Topographic Information in BCI: Preliminary Results on Stroke Patients
}

\author{
Andrea Biasiucci, Ricardo Chavarriaga, Benjamin Hamner, Robert Leeb, Floriana Pichiorri, \\ Fabrizio De Vico Fallani, Donatella Mattia and José del R. Millán
}

\begin{abstract}
Non-Invasive Brain-Computer Interfaces (BCI) convey a great potential in the field of stroke rehabilitation, where the continuous monitoring of mental tasks execution could support the positive effects of standard therapies. In this paper we combine time-frequency analysis of EEG with the topographic analysis to identify and track task-related patterns of brain activity emerging during a single BCI session. 6 Stroke patients executed Motor Imagery of the affected and unaffected hands: EEG sites were ranked depending on their discriminant power (DP) at different time instants and the resulting discriminant periods were used as a prior to extract EEG Microstates. Results show that the combination of these two techniques can provide insights about specific motor-related processes happening at a fine grain temporal resolution. Such events, represented by EEG microstates, can be tracked and used both to quantify changes of underlying neural structures and to provide feedback to patients and therapists.
\end{abstract}

\section{INTRODUCTION}

Despite recent advances in science and prevention, stroke is still a serious health problem that involves around 20 million people worldwide per year and represents the third most common cause of death. $75 \%$ of this population will survive the vascular accident, but approximately 5 million individuals will be disabled by their stroke [1]. The residual motor impairment will limit these people's ability to engage meaningful occupations including self care, work and leisure. Consequently, the development of new techniques to support and drive a more efficient functional rehabilitation ranks among the primary interests of current research. Several approaches aimed at optimizing motor behavior to restore occupational performance have been developed by occupational therapists, stressing person's active participation. Interestingly, several studies provide hints on the positive effects of mental practice in adjunction to physical practice [2]. A growing body of literature also suggests that similarities exist in terms of neural activity between the state in which a motor action is imagined and the state of execution, resulting in the benefits seen in mental practice [3].

The use of Brain-Computer Interfaces (BCI) protocols to promote recovery of motor function by encouraging and guiding plasticity phenomena occurring after stroke (or more

This work was supported by European ICT Programme Project TOBI FP7-224631

A. Biasiucci, R. Chavarriaga, B. Hamner, R. Leeb, and J.d.R. Millán are with the Chair on Non-Invasive Brain-Machine Interaction, École Polytechnique Fédérale de Lausanne, Switzerland andrea.biasiucci@epfl.ch

F. Pichiorri, F. De Vico Fallani and D. Mattia are with the Laboratorio di Neurofisiopatologia, Fondazione Santa Lucia, Rome, Italy donatella.mattialfsl.it generally after brain injury) has been proposed [4]. Discussion is currently underway over several factors including: the extent to which patients have detectable brain signals that can support training strategies; which brain signal features are best suited for use in restoring motor functions and how these features can be used most effectively; and what are the most effective approaches for BCI aimed at improving motor functions (for instance, what guidance should be provided to the user to maximize training that produces beneficial changes in brain signals). Preliminary findings suggested that event-related EEG activity time-frequency maps of event-related EEG activity and their classification are proper tools to monitor MI related brain activity in stroke patients and to contribute to quantify the effectiveness of MI [5]. Preliminary studies on stroke patients using BCI found that the best signals appear over the ipsilateral (unaffected) hemisphere [6]. Finally, the idea that BCI technology can induce neuroplasticity has received remarkable support from the community based on invasive detection of brain electrical signals [4].

Non-Invasive Brain-Computer Interfaces normally monitor variations in specific frequency bands of spectral power associated with different mental tasks, such as motor imagery, to generate an output that can be used for communication and control of devices such as a virtual keyboard, a telepresence robot or a powered wheelchair [7], [4]. The use of nonparametric methods, such as FFT, restricts the frequency analysis of EEG patterns in the time scale of seconds, losing the good temporal resolution of this recording modality [8]. On the other hand, EEG-Microstates [9] represent an alternative imaging analysis method that preserves time resolution, providing topographies that remain stable for tens to hundreds of ms. In this paper we want to address the issue of tracking brain patterns during BCI-aided stroke rehabilitation combining a standard frequency analysis of EEG with a topographic analysis. Extracted information could be used to provide feedback about relevant taskrelated mental patterns to patients or practitioners during the therapeutic cycle.

This paper is structured as follows: in Section II we present the experimental protocol we used to record EEG data from stroke patients, Section III presents the techniques we used to extract EEG discriminant frequency information and EEG microstates. In Section IV we present the results of the combined discriminant-topographic analysis and Section V addresses the major findings and issues related to the topic of this paper and the future directions. 


\section{EXPERIMENTAL PROTOCOL}

Six Stroke patients suffering from left or right hemisphere lesions participated in the experiment at Fondazione Santa Lucia, Rome, Italy. The subject was comfortably seated in an armchair placed in a dimly lit room with her/his upper limbs on a desk, visible to her/him, with hand posture on a side view. A screen is positioned on the desk in front of the patient and she/he is provided with a visual feedback. Scalp EEG potentials are collected from 61 positions (according to an extension of the 10- 20 International System), bandpass filtered between 0.1 and $70 \mathrm{~Hz}$, digitized at $200 \mathrm{~Hz}$ and amplified by a commercial EEG system. Each session is divided in runs consisting of 30 trials, temporally determined by a cursor appearing in the low center of the screen and moving towards the top at constant velocity on a straight trajectory. Total trial duration is $9 \mathrm{~s}$; intertrial interval is 1.5 s. During rest trials (Fig. 1; rest trial timeline), the patient is simply asked to watch the cursors trajectory on screen. During motor task trials (Fig. 1; Movement trial timeline), a green rectangle appears on top of the screen (rectangles width is $100 \%$ of screen width, rectangle height equals to $57 \%$ approximately of screen length, occupying the last $4 \mathrm{~s}$ of cursor trajectory) and the patient is asked to start performing the cued motor task (motor execution/motor imagery, unaffected/affected hand) when cursor reached the green rectangle and to continue until the end of trajectory. Each run is dedicated to a different motor task. Two different motor tasks (A and B) are examined. Task A consists of tonic grasping movement, Task B is a tonic complete finger extension.Command sequence is randomized. Runs of the the EEG session include $15 \pm 1$ rest trials and $15 \pm 1$ motor trials (total 30). The EEG session starts with the unaffected hand: in the first run the patient is asked to move his hand (first run - Task A, second run - Task B); in the following 2 runs the patient is asked to imagine the same movements (1 run - Task A, 1 run - Task B). The second part of the session involved the affected hand: in this case, if execution is not possible at all, patient is just asked to attempt the movement. In the remaining of this tudy we focus only in the motor imagery tasks.

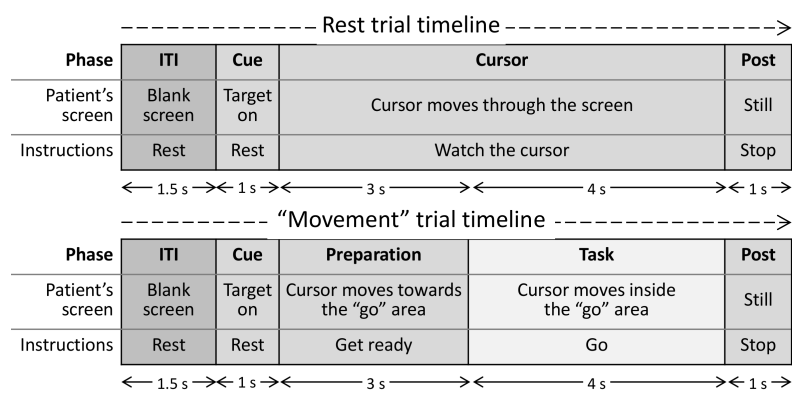

Fig. 1. Single trial description. Analysis for this study was constrained to the first 2 seconds of the "Go" window. "rest" trials were analyzed in the same time window.

\section{METHODS}

The techniques provided in this paper aim at combining a discriminant analysis with the topographic analysis of EEG signals. Briefly, discriminant analysis was used to select time windows within each run when EEG discriminant activity was localized over motor areas. Then, topographic analysis was performed on the selected window to assess whether the discriminant activity was reflected on changes in the duration or occurrence of the obtained microstates.

To do so, we first computed EEG Power Spectral Densities (PSD) for all electrodes and then ranked the contributions of all channels in all frequency bands through Canonical Variate Analysis (CVA) [10]. Furthermore, we considered different intervals of the spectrogram to characterize changes of discriminability in time. Then, the most informative time window was selected for further microstate analysis; we defined this procedure "time-constrained topographic analysis". EEG signals extracted from the informative window were clustered in topographic maps whose number of occurrences per second and average durations were then compared for every task (i.e. MI of the unaffected and affected hands movements - UH and AH respectively) against rest condition.

\section{A. Power Spectral Density Estimation \& Canonical Variate Analysis}

As a first step the 16 most external electrodes were discarded to avoid muscular contamination on the data (i.e. electrodes FPZ, AF7, AF8, F7, F8, FT7, FT8, T7, T8, TP7, TP8, P7, P8, PO7, PO8, Oz), then EEG signals were downsampled to $128 \mathrm{~Hz}$ and referenced to the Common Average Reference (CAR) before estimating their power spectral density (PSD) in the band $4-28 \mathrm{~Hz}$ with $4 \mathrm{~Hz}$ resolution over a window of 2 seconds from trigger onset. The PSD was computed every $50 \mathrm{~ms}$ using the Welch method with 5 overlapped $(25 \%)$ Hanning windows of $500 \mathrm{~ms}$.

Following previous studies, we computed the Discriminant Power (DP) of each feature using Canonical Variate Analysis [10]. For this study, we were interested in using this frequency analysis to determine when salient motorrelated EEG features were more likely to appear in time. Consequently, we extracted the DP information of nonoverlapping spectrogram blocks lasting $500 \mathrm{~ms}$. For every block, we computed the most discriminant electrodes for all frequency bands as the ones having a value equal or greater than $70 \%$ of the DP maximum and checked whether activity in the motor areas (i.e. electrodes FC5, FC3, FC1, FCz, FC2, $\mathrm{FC} 4, \mathrm{FC} 6, \mathrm{C} 5, \mathrm{C} 3, \mathrm{C} 1, \mathrm{Cz}, \mathrm{C} 2$, C4, C6, CP5, CP3, CP1, CPZ, CP2, CP4, CP6) was highly discriminant. We then picked the most discriminant electrodes covering motor areas from every time frame and we compared which of those showed the highest DP value. The time window associated with this most discriminant activity was finally selected for microstate analysis.

\section{B. EEG Microstates}

Epochs were extracted from each subject depending on the salient time window selected through the discriminant 
analysis described above. In the case of no discriminant pattern covering motor areas epochs were extracted in the interval from 0 to $2 \mathrm{~s}$ after trigger onset. EEG microstates were computed from concatenated epochs of MI condition and resting with a modified version of the $\mathrm{k}$-means clustering algorithm [11]. To extract $k$ microstates, this algorithm used the time-domain EEG signal, took each time instant as a 64-dimensional vector, and then clustered the time instances based on their vector orientations. It is initialized with $k$ 61-dimensional unit vectors of random orientation, and then alternates between a cluster-assignment phase and a dictionary-update phase until convergence is reached. In the cluster-assignment phase, each time instance was assigned to the dictionary element with the maximal magnitude cosine similarity. In the dictionary-update phase, for each dictionary element, the sum of the self-outer products of the associated time signals was taken, and the dictionary element was updated to the normalized dominant eigenvector of this matrix. We extracted a number of $k=5$ maps per subject and condition.

Once the set of microstates was learned, the time-domain signal was clustered with an additional penalty to encourage smoothness of the resulting signal. Following the literature [11], we used values of the window size $b=3$ and smoothness penalty $\lambda=5$. The smoothed microstates signal allowed for measurements of the occurrences and duration of the microstates that were not corrupted by random fluctuations in the EEG signal.

The number of map occurrences per second and mean duration (ms) of each microstate were calculated and compared to the occurrences and duration of those from the respective rest trials. Percentage variations (MI vs rest), were computed as follows:

$$
\left(\Lambda_{\text {Map }, M I}-\Lambda_{\text {Map,rest }}\right) / \Lambda_{\text {Map,rest }} \cdot 100,
$$

where $\Lambda$ represents either map occurrences or mean duration.

Obtained maps for one of the subjects are shown on Fig. 2. EEG Microstates provide physiologically relevant maps [12] that range from a motor-related lateralization (Fig. 2 map 3) to muscular artifacts such as eye blinks (Fig. 2 - map 4).
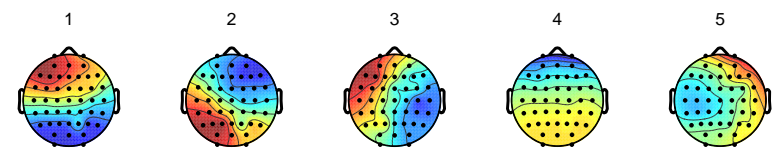

Fig. 2. Extracted microstates for Subject S6 (lesion on left hemisphere) performing MI of grasping movements (Task A) of the $\mathrm{AH}$.

\section{RESULTS AND DISCUSSION}

The discriminant analysis identified different combinations of electrode locations and frequencies as the peak of most discriminant activity in the four considered time frames from 0 to 2 s. Table I provides, for every subject and condition, the electrode, frequency and time frame containing the most
TABLE I

DISCRIMINANT ANALYSIS RESULTS (AFFECTED AND UNAFFECTED HAND: AH, UH RESPECTIVELY)

\begin{tabular}{|c|c|c|c|c|}
\hline Sub.(Lesion Site) & Task & Electrode & Freq. $[\mathrm{Hz}]$ & Time Win. $[\mathrm{s}]$ \\
\hline \multirow{4}{*}{ S1(L) } & AH B & & - & \\
\hline & $\mathrm{AH} \mathrm{A}$ & $\mathrm{CPz}$ & 16 & $0.5-1$ \\
\hline & UH B & $\mathrm{CP} 2$ & 16 & $0.5-1$ \\
\hline & & $\mathrm{C} 3$ & 12 & $0-0.5$ \\
\hline \multirow{4}{*}{ S2(R) } & $\overline{\mathrm{AH} \mathrm{B}}$ & $\mathrm{CPz}$ & 12 & $0-0.5$ \\
\hline & $\mathrm{AH} \mathrm{A}$ & $\mathrm{C} 6$ & 28 & $1-1.5$ \\
\hline & UH B & $\mathrm{C} 2$ & 24 & $0-0.5$ \\
\hline & UH A & FC6 & 28 & $0-0.5$ \\
\hline \multirow{4}{*}{ S3(L) } & $\overline{\mathrm{AH} \mathrm{B}}$ & 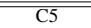 & 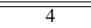 & $\overline{00-0.5}$ \\
\hline & $\mathrm{AH} \mathrm{A}$ & FC6 & 16 & $0-0.5$ \\
\hline & UH B & $\mathrm{CPz}$ & 4 & $1.5-2$ \\
\hline & UH A & $\mathrm{C} 3$ & 20 & $0-0.5$ \\
\hline \multirow{4}{*}{ S4(R) } & $\mathrm{AH} \mathrm{B}$ & & & \\
\hline & $\mathrm{AH} \mathrm{A}$ & FC4 & 16 & $0-0.5$ \\
\hline & UH B & $\mathrm{FC} 2$ & 4 & $0.5-1$ \\
\hline & UH A & $\mathrm{C} 2$ & 28 & $0.5-1$ \\
\hline \multirow{4}{*}{ S5(R) } & AH B & $\mathrm{FCz}$ & 8 & $0-0.5$ \\
\hline & $\mathrm{AH} \quad \mathrm{A}$ & $\mathrm{Cz}$ & 16 & $0.5-1$ \\
\hline & UH B & $\mathrm{C} 1$ & 12 & $0-0.5$ \\
\hline & UH A & $\mathrm{CPz}$ & 16 & $1-1.5$ \\
\hline \multirow{4}{*}{ S6(L) } & $\overline{\mathrm{A} \mathrm{AH} \mathrm{B}}$ & $\overline{\mathrm{C} 1} 1$ & 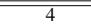 & "0.5-1 \\
\hline & $\mathrm{AH} \mathrm{A}$ & - & - & - \\
\hline & UH B & - & - & - \\
\hline & & $\mathrm{C} 3$ & 28 & $0-0.5$ \\
\hline
\end{tabular}

discriminant information. Reported spatial locations and frequency are compatible with current BCI literature for MI tasks. In four of the analyzed conditions (namely S1 - AH A, S4 - AH B, S6 - AH A, S6 UH B), no discriminant activity was found over motor areas. It is worth noticing that three of these cases correspond to motor imagery of the affected hand.

Concerning the time-constrained topographic analysis, Table II provides the percentage variation in terms of occurrences per second and average map durations, for every subject and condition. Note that, since microstates are extracted independently for every condition, no comparison can be made column-wise. Variations in map occurrence were consistently larger than changes in average map duration. The maps associated with the two strongest variations in occurrence for every conditions are highlighted on Table II. Remarkably, maps that exhibit the largest occurrence variation show a common lateralized trend in at least one of the two selected with this simple rule, and this was observed for most of the subjects. Values "N/A" refer to maps that, after the smoothing described in Section III, do not occurr at all in the selected window.

Since the topographic analysis was performed on the time windows obtained from the discriminant analysis, this suggests that the maps that exhibit large variations are related to task-specific EEG topographies. This is particularly important for the case of stroke rehabilitaton, since it is possible to continously monitor the generation of topographic maps with a fine temporal resolution.

\section{CONCLUSIONS}

This study aims at combining standard BCI discriminant analysis with microstate extraction in order to identify potential EEG topographies related to motor imagery. The use of a discriminant framework allows us to constrain the analysis on a relevant time window related to motor imagery only. Furthermore, the use of EEG microstates can capture short, 
TABLE II

TOPOGRAPHIC ANALYSIS - OCCURRENCES (DURATION)

\begin{tabular}{|c|c|c|c|c|c|c|}
\hline & Task & \multicolumn{5}{|c|}{$\%$ Variation, MI vs rest } \\
\hline & & Map 1 & Map 2 & Map 3 & Map 4 & Map 5 \\
\hline \multirow{4}{*}{$\mathrm{S} 1(\mathrm{~L})$} & $\mathrm{AH} \quad \mathrm{B}$ & $41(34)$ & $-11(-23)$ & $81(30)$ & $-77(-29)$ & N/A \\
\hline & $\mathrm{AH} \mathrm{A}$ & $0(-8)$ & $-24(-5)$ & $18(10)$ & 35 (14) & N/A \\
\hline & UH B & $-6(-17)$ & $-88(-48)$ & 417 (191) & $33(-7)$ & N/A \\
\hline & UH A & $1(-49)$ & $15(21)$ & $404(42)$ & 100 (11) & N/A \\
\hline \multirow{4}{*}{$\mathrm{S} 2(\mathrm{R})$} & $\overline{\mathrm{AH} \quad \mathrm{B}}$ & $45(66)$ & $-32(-19)$ & $16(-3)$ & $22(50)$ & $-38(-35)$ \\
\hline & $\mathrm{AH} \mathrm{A}$ & $-13(11)$ & $12(-8)$ & $53(15)$ & $-57(-39)$ & $200(36)$ \\
\hline & UH B & $6(-18)$ & $-8(-22)$ & $22(3)$ & $71(-6)$ & $0(-1)$ \\
\hline & UH A & $8800(100)$ & $-60(-57)$ & $-36(-1)$ & 44 (15) & $800(108)$ \\
\hline \multirow{4}{*}{ S3(L) } & AH B & $0(30)$ & $-1(10)$ & $8(-13)$ & $-48(-22)$ & $-87(-18)$ \\
\hline & $\mathrm{AH} \mathrm{A}$ & $2(-32)$ & $34(98)$ & $-99(-89)$ & N/A & N/A \\
\hline & UH B & $-19(-9)$ & $-35(-30)$ & 63 (12) & $53(1)$ & 205 (29) \\
\hline & UH A & $40(42)$ & $9(12)$ & $-66(-28)$ & $-2(4)$ & $-56(-12)$ \\
\hline \multirow{4}{*}{ S4(R) } & AH B & $-4(308)$ & $-58(-18)$ & $-80(-44)$ & $-62(-31)$ & N/A \\
\hline & $\mathrm{AH} \mathrm{A}$ & $3(-27)$ & $7(-3)$ & 73 (37) & $-10(-25)$ & N/A \\
\hline & UH B & $0(14)$ & $29(7)$ & $131(35)$ & $-36(-27)$ & $-57(-23)$ \\
\hline & UH A & $-13(49)$ & $-51(-17)$ & $-17(0)$ & $17(-3)$ & $51(6)$ \\
\hline \multirow{4}{*}{ S5(R) } & AH B & $46(32)$ & $-6(7)$ & $19(25)$ & $89(43)$ & $-78(-45)$ \\
\hline & $\mathrm{AH} \mathrm{A}$ & $27(50)$ & $14(-8)$ & $90(14)$ & $-80(-51)$ & 165 (37) \\
\hline & UH B & $-3(9)$ & 9 (15) & $-45(-2)$ & $-5(-5)$ & N/A \\
\hline & UH A & $11(9)$ & $70(48)$ & $-48(-18)$ & $3(-14)$ & $-53(-27)$ \\
\hline \multirow{4}{*}{ S6(L) } & AH B & $3(0)$ & $61(12)$ & $-48(-18)$ & $28(4)$ & $-2(-11)$ \\
\hline & $\mathrm{AH} \mathrm{A}$ & $-10(-32)$ & $-7(-7)$ & $159(44)$ & $-39(-11)$ & $110(9)$ \\
\hline & UH B & $-33(-39)$ & $-12(-17)$ & $94(40)$ & $42(15)$ & 124 (15) \\
\hline & UH A & 73 (37) & $5(9)$ & $-23(-2)$ & $-25(-14)$ & $-59(-24)$ \\
\hline
\end{tabular}

transient and stable voltage configurations on the scalp, thus providing insights on underlying mental processes.

Given the fact that selected microstates have been extracted and analyzed in the most discriminant time frame in terms of modulation of motor-related rhythms [13], they are very likely to be related to the short and transient mental processes associated with the motor imagery of the affected and unaffected hand. We believe that the results we present in this paper strongly motivate further analysis in this direction, especially correlating functional recovery to $\mathrm{BCI}$ performance.

The time-constrained topographic analysis shows that changes in maps occurrence, rather than in their average duration, are more significant when comparing MI against rest. This suggests that the frequency of appearance of particular maps may be a good indicator of proper execution of the rehabilitation tasks. Consequently, it could be possible to use this measure to provide online feedback for therapists in order to support the rehabilitation process. This will be particularly suited for the applications related to stroke treatments.

As previously proposed [14], the use of combined sessions of standard therapy and BCI-aided rehabilitation can serve as a way to facilitate recovery through mental rehearsal. In addition, proposed techniques represent an imaging modality to monitor long-term changes in produced patterns representing cortical reorganization.

We are currently working on an extension of this study considering, for the same set of patient, more sessions recorded in the framework of a rehabilitation cycle.

\section{REFERENCES}

[1] S. MacMahon, "Introduction: The global burden of stroke", Clinician's Manual on Blood Pressure and Stroke Prevention, J. Chalmers Science

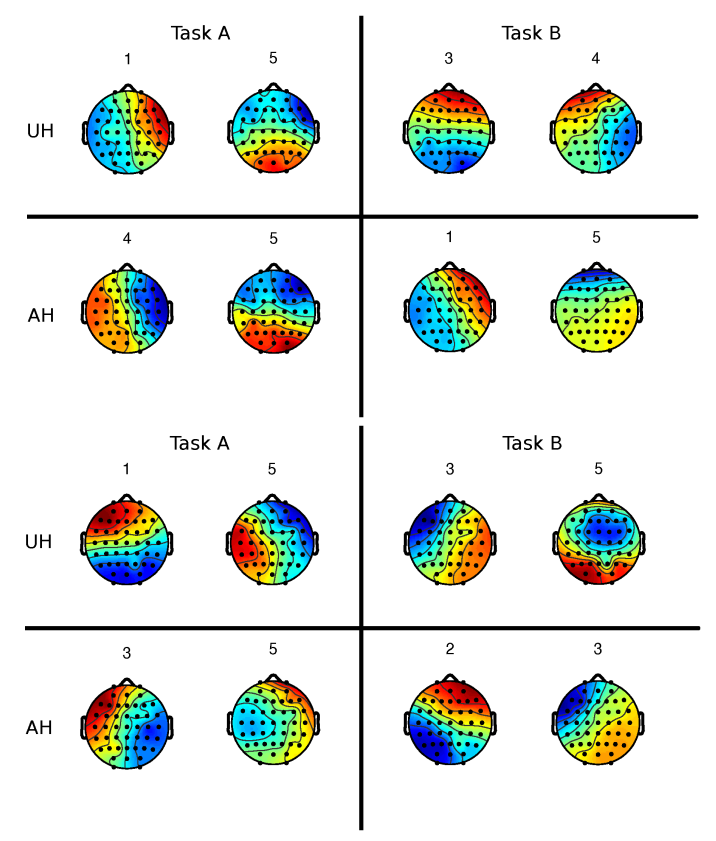

Fig. 3. Maps associated with strongest changes in occurrences (see Table II for details) for Subjects S2 (top) and S6 (bottom).

Press London, 2002, pp. 1-6.

[2] D.M. Nilsen, G. Gillen and A.M. Gordon, "Use of mental practice to improve upper-limb recovery after stroke: A systematic review", The American Journal of Occupational Therapy, vol. 64(5), 2010.

[3] M. Jeannerod, "Neural simulation of action: A unifying mechanism for motor cognition", NeuroImage, vol. 14, 2001, pp. S104-S109.

[4] J. del R. Millán, R. Rupp, G. R. Müller-Putz, R. Murray-Smith, C. Giugliemma, M. Tangermann, C. Vidaurre, F. Cincotti, A. Kübler, R. Leeb, C. Neuper, K.-R. Müller and D. Mattia, "Combining braincomputer interfaces and assistive technologies: State-of-the-art and challenges", Frontiers in Neuroscience, vol. 4, 2010.

[5] R. Scherer, A. Mohapp, P. Grieshofer, G. Pfurtscheller and C. Neuper, "Sensorimotor EEG patterns during motor imagery in hemiparetic stroke patients", Int. J. Bioelectromag, vol. 9, 2007 pp. 155-162.

[6] E. Buch, C. Weber, L.G. Cohen, C. Braun, M.A. Dimyan, J. Mellinger, A. Caria, S. Soekadar, A. Fourkas and N. Birbaumer,"Think to move: A neuromagnetic brain-computer interface (BCI) system for chronic stroke". Stroke, vol. 3

[7] J.R. Wolpaw, N. Birbaumer, D.J. McFarland, G. Pfurtscheller and T.M. Vaughan, "Brain-computer interfaces for communication and control", Clinical neurophysiology, vol. 113(6), 2002, pp. 767-791.

[8] T. Koenig, F. Marti-Lopez and P. Valdes-Sosa, "Topographic timefrequency decomposition of the EEG", NeuroImage, vol. 14(2), 2001.

[9] D. Lehmann, "Multichannel topography of human alpha EEG fields", Electroenceph. and Clinical Neurophysiology, vol. 31(5), 1971.

[10] F. Galán, P.W. Ferrez, F. Oliva and J. Guàrdia and J. del R. Millán "Feature extraction for multi-class BCI using canonical variates analysis", IEEE Int. Symposium on Intelligent Signal Processing, 2007.

[11] R.D. Pascual-Marqui, C.M. Michel, D. Lehmann, "Segmentation of brain electrical activity into microstates: Model estimation and validation”, IEEE Trans. on Biomedical Engineering, vol. 42(7), 1995.

[12] M.M. Murray, D. and Brunet and C.M. Michel, "Topographic ERP analyses: A step-by-step tutorial review", Brain Topography, vol. 20(4), 2008 pp. 249-264.

[13] G. Pfurtscheller, C. Brunner, A. Schlögl and F.H. Lopes da Silva, "Mu rhythm (de) synchronization and EEG single-trial classification of different motor imagery tasks", NeuroImage, vol. 31(1), 2006.

[14] N. Sharma, V.M. Pomeroy, J.C. Baron , "Motor imagery: A backdoor to the motor system after stroke?", Stroke, vol. 37(7), 2006, pp. 19411952. 